\title{
Research on Maturity Evaluation Mechanism of Video Teaching in College English Teaching based on BP Neural Network Algorithm
}

\author{
Jing Liao \\ Wuhan Business University, Wuhan, Hubei, 430056
}

Keywords: Maturity Evaluation; video teaching; neural network

\begin{abstract}
There are many evaluation methods of classroom teaching quality. Based on the analysis of the traditional evaluation methods, this paper puts forward the application of BP neural network theory in the evaluation of college classroom teaching quality. It not only solves the problems of qualitative indicators and quantitative indicators in the comprehensive evaluation index system, but also effectively overcomes the problems of establishing complex mathematical models and mathematical analytical expressions in the traditional evaluation process, and also avoids artificial subjective randomness, making assessment more effective and accurate, so as to open up a new method for the reasonable evaluation of classroom teaching quality.
\end{abstract}

\section{Introduction}

In order to meet the requirements of the current new situation, higher education in China needs to constantly improve and upgrade its own level of development through reforms. In higher education, colleges and universities are the main places for imparting knowledge and culture. The teaching classroom of university teachers is the main carrier for this goal. Therefore, colleges and universities put forward higher requirements for the teaching level of teachers. It is necessary to adopt various measures to improve teachers' teaching quality. At present, many colleges and universities use teaching methods that are graded by students. This method divides all the school's courses into several major categories, such as theoretical courses, internships, and experimental courses. It sets some evaluation indicators for each type of course. The students will anonymously score the teacher of the course they are studying based on these indicators. At the end of each semester, students' scores are summarized by the educational administration department or the university evaluation center to strengthen teaching management and enhance teachers' teaching quality. Although this method strengthens the school's management of teaching to a certain extent, the evaluation results also reflect the level of teachers' teaching to a certain extent, making teachers pay more attention to the students' feelings in the teaching process and help improve the teaching level of the teachers.

\section{Significance of Classroom Teaching Quality Evaluation}

The quality of higher education in China has always been the focus of attention. In recent years, the Ministry of Education has conducted dozens of colleges and universities to evaluate teaching quality every year. Therefore, the correct evaluation of teaching quality is also a hot topic in recent years. How to raise the level of higher education? The quality of education has become the focus of current higher education. In colleges and universities, classroom teaching is still the main channel for implementing education. Its quality reflects and determines the quality of education in universities to a large extent. Therefore, improving the quality of classroom teaching has become a top priority. Classroom teaching includes many factors such as teaching conditions, course difficulty, teacher's teaching, and learning effects. They interact to form a network of teaching functions. Among them, teacher's classroom teaching is the most important link, which determines the level of talent cultivation and influence. The quality of life of teachers and students. Therefore, the construction of a scientific, systematic, and effective classroom teaching quality evaluation system is of great significance for the improvement of classroom teaching evaluation, the expansion of 
university teacher management theory, and the improvement of classroom teaching quality.

The weighted average method is used by the school teaching management department to formulate various indicators for evaluation, and according to the importance of each indicator to set the weight of each indicator, the questionnaire is issued, so that the appraiser gives the scores of the indicators of the evaluated person. Then, a weighted sum is obtained to obtain the total score, and the grade of the score is used to judge the quality of the assessed person's teaching quality. Although the weighted average method is relatively simple, this method artificially assigns a simple linear relationship to each evaluation index, and gives the weight of each evaluation factor based on experience. Obviously, it cannot prove whether each evaluation factor has linear increase. Sex does not justify the weight.

The Analytical Hierar-chy Process (AHP method) proposed by A.L. Saaty, a US operational researcher in the 1970s, is a qualitative and quantitative decision analysis method. Analytic Hierarchy Process (AHP) Basic principle: First, the problem is hierarchized, and the problem is decomposed into an orderly level according to the nature of the problem and the overall goal. Then the experts are asked to make more objective judgments on the factors at each level. A quantitative representation of relative importance; then establish a mathematical model, calculate the weights of the relative importance of all factors at each level, and sort them; finally, according to the ranking results, make planning decisions and select solutions to the problem. Using the analytic hierarchy process A H P can get the weight of the evaluation factors, but it also requires a linear relationship between these evaluation indicators, otherwise it will affect the evaluation of the AHP model. Second, when multiple evaluation experts give different judgment matrices, which one is the actual ideal value structure, the AHP model cannot answer this question.

Fuzzy mathematics is the mathematics of studying and dealing with ambiguity phenomena. It was founded in 1965 by American cybernetics expert L.A.Zadeh. The principle of multi-level comprehensive evaluation of fuzzy mathematics is to first judge the multiple factors of the same thing, divide it into several major factors according to a certain attribute, and then conduct a comprehensive evaluation of a large factor at the initial level, and then on the basis of Hierarchical comprehensive evaluation of the results of high-level comprehensive evaluation. [3] The fuzzy comprehensive evaluation method is a method of using fuzzy mathematics to obtain a teacher's classroom teaching quality evaluation conclusion through the fuzzy mathematics comprehensive evaluation model. The key to its successful application lies in correctly defining the set of factors for fuzzy evaluation and constructing a fuzzy evaluation matrix. The fuzzy comprehensive evaluation method can be used to evaluate the value ranking or mutual preferential relationship of the objects to be evaluated. However, this method requires the establishment of a suitable evaluation matrix for the evaluation object, and this will result in different evaluation matrixes due to the inconsistency of different experts, making the final Inconsistent results of evaluation results.

\section{Feasibility of Neural Network Used in Teaching Quality Evaluation System}

As a powerful tool for studying nonlinear fitting and classification, neural networks have highlighted their superiority in pattern recognition, automatic control, and prediction. Based on the existing training data, the neural network can learn regular things from a large number of existing complex data through continuous learning and training, so as to achieve the purpose of exploring the unknown. It can handle any kind of data in particular, which is not achieved by many traditional methods, and therefore its accuracy is high. At the same time, it can also handle multiple inputs, taking into account the effect of each input on the output. Therefore, the use of neural networks in the evaluation system of university teaching quality not only solves the problems of qualitative indicators and quantitative indicators in the comprehensive evaluation index system, but also solves the complex modeling problems of traditional evaluation systems, and avoids the arbitrariness of artificial customers. Guaranteed effective evaluation results.

The BP neural network is a widely used neural network that corrects model weights and thresholds by backpropagating errors [8]. When the BP neural network is used in the evaluation of college teaching quality, each indicator in the comprehensive evaluation system of teaching quality 
is taken as a neuron input, and the final result of the evaluation is output as an output, thereby establishing an evaluation model. During the training process, if there is an error between the output magnitude and the expected magnitude and exceeds the specified range, the connection weights and the hidden layer and output layer nodes between the layers are adjusted according to the error transmission method. The threshold, until the system error is controlled within an acceptable range, the training is stopped [9]. The weights and thresholds at this time will not change anymore. The resulting network is a correct representation of adaptive learning. A trained neural network can be used as an effective tool for qualitative and quantitative integration for objects other than training data. Correct evaluation.

\section{Classroom Teaching Quality Evaluation Method Based on BP Neural Network}

An artificial neural network (A N N) is an information processing system or computer based on the structure and function of a biological brain, abbreviated as a neural network, abbreviated as ANN (Artificial Neural Network). BP neural network is a multi-layer feed-forward network. At present, in the practical application of artificial neural network, most of the neural network models are based on the BP algorithm or its variations. The novelty of the BP neural network lies in its ability to model nonlinear processes without understanding the origin of the data. It has the characteristics of nonlinear mapping, learning classification, and real-time optimization. Therefore, it is used for pattern recognition, nonlinear classification, etc. Open up new ways. According to this feature, this paper intends to use BP neural network theory to establish the model of teaching quality evaluation system. After training the network, it can evaluate the teaching quality in a graded manner, thus providing a meaningful reference value for the study of teaching quality evaluation.

The evaluation index of classroom teaching quality itself has a guiding effect on teaching, that is, what indicators are evaluated and what indicators the teacher will value. Therefore, the establishment and selection of indicators is extremely important. In China, there is no strict definition of the evaluation index system and it is generally formulated by the school education department. Since it is difficult to compare the teaching of different subjects, different nature courses, different teaching links, and different teaching subjects, the consideration of the teaching quality evaluation system is mainly based on the most basic factors that can directly reflect the quality of teaching and have commonalities. . According to the indicators required by the Ministry of Education for the evaluation of undergraduate higher education, after comprehensive analysis, this paper sorted out the evaluation index system of classroom teaching quality based on students.

Because a three-layer BP network can approximate any mapping relationship with arbitrary precision, in order to reduce the consumption of memory resources and improve the learning rate of the network, this paper adopts a three-layer BP network structure to construct an evaluation model, which is both input layer and implicit. Layer, output layer. The number of neurons in the input and output layers of the BP neural network is determined by the dimensions of the input and output vectors. The dimension of the input vector is the number of factors that affect the quality of classroom teaching. Therefore, according to Table 1, the number of neurons at the input layer is 12 . The result of teaching evaluation is only one, so the number of output layers is 1 , and the range of values is $[0,1]$. The transfer function of the output layer neurons uses the Sigmoid function. Using trial and error method to get the best number of hidden layer nodes is 7. For the activation function on the hidden layer unit, select the $\mathrm{t}$ a $\mathrm{n} \mathrm{s}$ i g hyperbolic tangent function. The BP network learning algorithm selects the LMBP optimization algorithm, and the LMBP optimization algorithm is an improvement over the traditional learning algorithm, and the convergence speed and accuracy are better.

The omni-directional evaluation system refers to the work performance of teachers in all aspects including the leadership department, the steering group, colleagues, teachers, and students. Through this kind of performance evaluation, the evaluated teachers can obtain multi-angle feedback from superior leaders, supervisors, themselves, and students, so that they can more clearly understand their own shortcomings and strengths and provide basis for later development and career planning. 
Facts have proved that this all-round evaluation system is comprehensive and effective. The comprehensive evaluation system of university teaching quality includes a teaching evaluation system and a learning evaluation system. The evaluation target in the evaluation system is college teachers. The evaluation subjects include supervisors, peers, students, and teachers themselves. In the evaluation system for learning, the object of evaluation is the student. The subject of evaluation is the supervisor, the teacher, other students and the student himself. The indicators of the teaching evaluation system include teachers' mutual evaluation, teachers' self-evaluation, supervision and evaluation, and student's evaluation of teaching. The indicators of the learning evaluation system include peer evaluation, student self-evaluation, supervision evaluation, and teacher evaluation. The indicators link each other and influence each other to form an all-around college teaching quality evaluation system.

\section{Conclusion}

This paper constructs an all-around teaching quality evaluation system, establishes a teaching quality evaluation index system based on the requirements of different universities for teaching quality, and constructs a two-level neural network system based on the seven secondary indicators under the two systems, and trains them separately. The training results show that: The network can fit the training data well and the prediction effect is significant. Through the neural network, a reasonable evaluation of the teaching quality of the college teachers overcomes the direct influence of a single human factor on the evaluation results, and provides a new scientific and reasonable method for the evaluation of college teaching quality, which provides a beneficial effect on the research and development of teaching quality assessment. The reference.

\section{References}

[1] Zhao Guozhu, Li Zhiru, Jin Yi. Analysis of the problem of teaching quality evaluation in colleges and universities [J]. Education and Teaching Forum, 2013 ( 5): 263-265.

[2] He Qiang. The Improvement and Exploration of Academic Evaluation of Teaching in Universities [J]. Jiangsu Higher Education, 2010, (4): 58 - 60.

[3] Zhong Chunhua, Han Shengjuan. An Empirical Study on the Influencing Factors of Non-teaching of Students' Evaluation of Teaching [J]. Chinese Adult Education, 2012 (13): 118-119.

[4] Zhang Xiangdong, Zhang Shukun, Zhang Qi. Some thoughts on the evaluation of teachers' teaching quality [J]. Journal of Dalian University, 2009, 30( 4) : 114-117.

[5] Zheng Yanfu. Study on Teaching Quality Evaluation of Undergraduate College Teachers [J]. Xuzhou: China University of Mining and Technology, 2012. 